\title{
Comparaison de types génétiques de poules pour une production d'œufs locale : F1 (Fayoumi $x$ Leghorn) et croisement terminal ISA au Maroc
}

\author{
K Benabdeljelil ${ }^{1}$, P Mérat ${ }^{2}$ \\ 1 Institut agronomique et vétérinaire Hassan-ll, département des productions animales, \\ BP 6202, Rabat-Instituts, Rabat, Maroc; \\ 2 INRA, laboratoire de génétique factorielle, 78352 Jouy-en-Josas cedex, France
}

(Reçu le 28 avril 1994; accepté le 11 octobre 1994)

\begin{abstract}
Résumé - Un croisement de $1^{\text {re }}$ génération entre une lignée de la race Égyptienne Fayoumi et une lignée expérimentale a été comparé à l'Institut agronomique et vétérinaire Hassan-II (Rabat, Maroc) à un croisement commercial de type "pondeuse à œuf brun" (ISA) pour la production d'œufs en batteries dans un bâtiment d'un type courant au Maroc (en dur avec fenêtres et sans isolation thermique particulière). Le pourcentage de ponte jusqu'à l'âge de 70 sem différait peu entre les types génétiques comparés. En revanche, le poids moyen de l'œuf était de $10 \mathrm{~g}$ inférieur pour le croisement expérimental et l'efficacité alimentaire de ce croisement était inférieure à celle du croisement commercial. Les qualités des œufs (densité, pourcentage de coquilie, unités Haugh) ne diffèrent pas entre les croisements comparés, à l'exception du pourcentage de jaune, plus élevé dans le croisement Fayoumi x Leghorn. Le poids corporel des poules adultes était voisin pour les 2 types génétiques.
\end{abstract}

poule / production d'œufs / type génétique / Maroc

Summary - Test of genetic types for local egg production in Morocco: F1 (Fayoumi x Leghorn) and ISA terminal cross. $A$ F1 between a line of the Egyptian breed Fayoumi and an experimental Leghorn line was compared at the agricultural and veterinary Hassan-II Institute (Rabat, Morocco) with a commercial cross of a brown-egg layer type (ISA) for egg production in batteries, in a windowed uninsulated house of a classical type in Morocco. The laying percentage until the age of 70 weeks differed little between genetic types. Conversely, the mean egg weight was $10 \mathrm{~g}$, inferior for the experimental cross and the feed efficiency of this cross was inferior to that of the commercial cross. The egg qualities (density, percentage of shell, Haugh units) did not differ between the compared crosses, except the percentage of yolk, which was higher for the Fayoumi $x$ Leghorn cross. The adult body weight of hens was similar for the 2 genotypes.

\section{hen / egg / production / genetic type / Morocco}




\section{INTRODUCTION}

Nous avions précédemment présenté (Benabdeljelil et Mérat, 1992) plusieurs comparaisons de croisements expérimentaux ou commerciaux dans les conditions locales du Maroc, notamment, pour la production d'œuis, un croisement réunissant les gènes $d w$ (nanisme lié au se ..) et $\mathrm{Na}$ (cou nu), avec un croisement commercial de type "œuf brun». Les 2 gènes mentionnés étaient supposés contribuer à une meilleure adaptation aux conditions locales.

L'objectif du présent travail était de comparer les performances d'élevage et de production d'œufs d'un croisement F1 (Fayoumi $x$ Leghorn) entre une lignée d'origine Égytienne et une lignée Leghorn expérimentale, avec un croisement commercial de type œuf brun (ISA-Brown). La recherche d'une adaptation aux conditions d'élevage du Maroc passait ici par l'utilisation d'une lignée locale comme l'un des parents du croisement $F 1$ testé.

\section{MATÉRIEL ET MÉTHODES}

\section{Génotypes - conditions d'élevage}

La lignée de la race Égyptienne Fayoumi était conservée au laboratoire de génétique factorielle. Elle provenait d'une lignée antérieurement sélectionnée à l'université du Caire dont un échantillon avait été envoyé en 1979 à Jouy-en-Josas. La lignée Leghorn était également conservée au laboratoire de génétique factorielle. Elle était en ségrégation pour le gène de nanisme lié au sexe $d w$ et provenait d'un croisement d'une lignée Leghorn naine reçue en 1974 de l'Institut für Kleintierzucht (Celle, Rép féd d'Allemagne) et de coqs Leghorn Babcock (croisement terminal) fournis par l'ISA en 1991.

Le croisement était fait dans le sens mâle Fayoumi $x$ femelle Leghorn de façon à n'obtenir que des filles de taille normale $\left(D w^{+}\right)$. L'éclosion avait lieu le 9/11/91 et les poussins étaient expédiés à Rabat par avion en même temps qu'un lot de femelles du croisement commercial ISABrown.

Les conditions d'élevage et celles de la phase de ponte étaient semblables à celles décrites dans la série expérimentale précédente (Benabdeljelil et Mérat, 1992).

Les poulettes étaient élevées dans une poussinière avec fenêtres, au sol sur litière de paille, la densité étant légèrement inférieure à $10 / \mathrm{m}^{2}$. Chaque type génétique était réparti dans une loge séparée. Un aliment commercial "croissance" était donné ad libitum à tous les poussins jusqu'à l'âge de 8 sem, puis un aliment cpoulettes" de 8 à 20 sem, avec, pour les poulettes ISA, in rationnement "skip-a-day".

Les poussins étaient vaccinés contre la maladie de NewCastle, la bronchite infectieuse et la maladie de Gumboro, la variole, l'encéphalomyélite et plusieurs maladies parasitaires.

L'éclairement, de $24 \mathrm{~h}$ par jour les 3 premiers j, passair ensuite à $14 \mathrm{~h} 30$, puis les poulettes étaient graduellement amenées à $16 \mathrm{~h} 30$ selon le programme lumineux adapté au croisement ISA. L'intensité lumineuse correspondait à une puissance de $4 \mathrm{~W} / \mathrm{m}^{2}$.

Les poulettes, au nombre de 60 par type génétique, étaient transférées en cages de ponte à l'âge de $18 \mathrm{sem}$. Ces cages, d'un type commercial, contenaient chacune 3 poules du même type.

L'aliment donné aux pondeuses, en farine, contenait $16,5 \%$ de matières azotées, $2850 \mathrm{Kcal}$ d'énergie métabolisable $/ \mathrm{kg}$ et $3,5 \%$ de calcium. Après le pic de ponte, les poules ISA étaient rationnées à $120 \mathrm{~g} / \mathrm{j}$ environ (moyenne par poule), l'autre croisement continuant à recevoir l'aliment ad libitum. Cette procédure correspondait aux recommandations courantes pour les poules ISA pour éviter leur augmentation de poids ; elle ne pouvait a priori les pénaliser, ne comportant d'ailleurs qu'une restriction légère.

\section{Mesures et analyses}

Le pourcentage de ponte par périodes est basé sur les poules présentes durant chaque période. Le poids moyen des œufs porte sur tous les œufs de chaque poule durant 3 j consécutifs dans la période correspondante. La masse d'œufs est estimée comme le produit du pourcentage de ponte par le poids moyen des œufs. L'indice de 





consommation est déduit de la consommation journalière et de la masse d'œufs. Les caractéristiques de qualité des œufs sont mesurées sur $3 œ u t s$ consécutifs par poule pour chaque période. Les coquilles ont été lavées, puis séchées à température ambiante et l'épaisseur a été déterminée avec les membranes coquillières à l'aide d'un micromètre dans la zone équatoriale de l'œuf. La densité a été déterminée par flottation dans les solutions salines $(\mathrm{NaCl})$ de concentration croissant de 0,005 allant de 1,065 à 1,100 .

Pour chaque caractère et chaque période les deux croisements étaient comparés par un test $t$.

\section{RÉSULTATS ET DISCUSSION}

Les tableaux I, II, III présentent la comparaison entre types génétiques (croisement Fayoumi $x$ Leghorn et (SA commercial) pour les principaux caractères mesurés à divers âges.

Le type commercial bénéficie d'une sélection intensive depuis des générations, qui doit a priorilui donner un avantage pour les principaux caractères de productivité par rapport à un croisement faisant appel à une lignée expérimentale non intensivement sélectionnée (Leghorn) et à une lignée locale (Fayoumi). Le croisement de ces 2 lignées peut, en revanche, être supposé relativement favorisé en conditions climatiques chaudes, et peut-être vis-à-vis de certains facteurs de stress ou agents pathogènes locaux, éventuellement aussi pour des critères de qualité des produits (œufs). Le croisement $F 1$ peut aussi posséder un hétérosis dont il fallait évaluer l'importance.

En ce qui concerne les conditions d'environnement, l'état sanitaire était dans l'ensemble satisfaisant et des différences très importantes à cet égard ne pouvaient donc s'extérioriser. Quant à la température, elle présentait des écarts relativement modérés durant la phase de ponte contrôlée. D'autre part, les conditions d'élevage et de logement comportaient des bâtiments "en dur» et, pour la ponte, des cages et ne s'éloignaient que modérément aussi des conditions industrielles «standard». Cela peut expliquer en particulier que le taux de mortalité cumulé en phase de ponte n'ait pas été inférieur pour la $F 1$ avec la race locale comparée au type commercial. II a même été supérieur, non significativement cependant. Entre les âges 43 et 70 sem, 7 poules ISA et 17 poules F1 (Fayoumi $x$ Leghorn) sont mortes, correspondant à un pourcentage respectif de 11,7 et $28,3 \%$. II semble que ceci a résulté, en tout ou partie, d'un cannibalisme plus fréquent dans la F1 Fayoumi x Leghorn.

Tableau II. Poids corporel à divers âges : F1 (Fayoumi x Leghorn) et ISA-Brown.

Âge

(sem)
Poids moyen ( $g$ )

Signification

Fayoumi $\times$ Leghorn
NS 


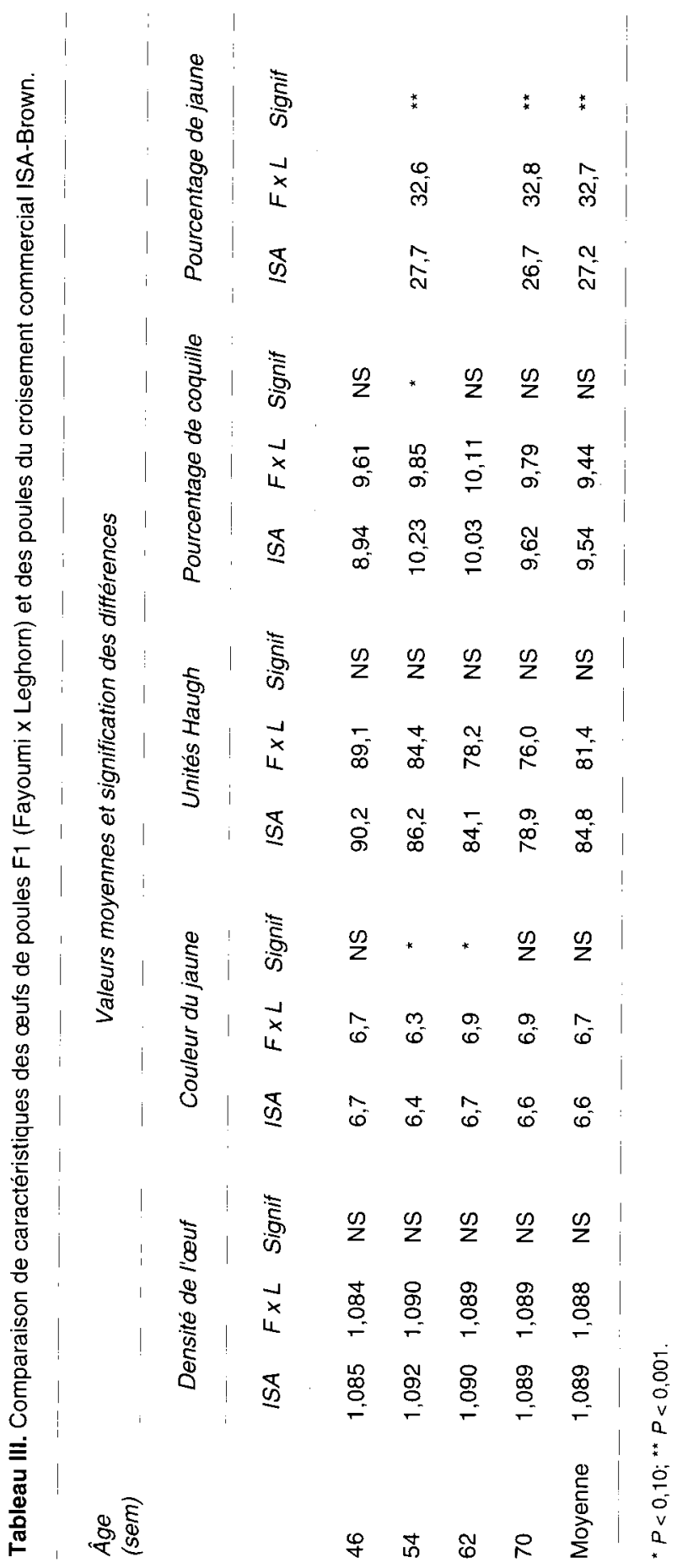


Quant aux performances de production (tableau I), elles ne font pas apparaître, dans l'ensemble, d'avantage substantiel du type commercial, du moins pour le pourcentage de ponte. Le niveau relativement satisfaisant du croisement expérimental pour ce critère dans ces conditions suggère un hétérosis appréciable dans ce croisement. Le poids corporel (tableau II) du croisement Fayoumi x Leghorn, par ailleurs, ne le désavantage pas a priori pour l'importance des besoins alimentaires d'entretien.

En revanche, à nombre d'œufs équivalent, le poids moyen de l'œuf, et en conséquence la masse d'œufs, est inférieur d'une dizaine de grammes pour le croisement expérimental, dont la consommation d'aliment par jour est cependant légèrement supérieure à celle du croisement ISA. Ce désavantage, dû surtout au poids d'œuf insuffisant apporté par le Fayoumi, entraîne une moindre efficacité alimentaire (tableau I). On peut cependant remarquer que, sur le marché local, le poids moyen de l'œuf a moins d'importance que dans les pays européens.

La qualité des œufs ne présente pas de différences très notables entre types génétiques, sauf toutefois le pourcentage de jaune, nettement plus élevé dans le croisement Fayoumi x Leghorn, que ne le fait prévoir la différence de poids moyen de l'œuf. Ce caractère, qui n'est habituellement pas pris en considération par les sélectionneurs, est apporté par la race Fayoumi qui est connue pour produire des œufs à pourcen- tage élevé de jaune (voir par exemple Mérat et al, 1983). En tant que liée à un plus fort taux de matière sèche dans l'œuf et aussi à un apport plus important d'acides gras essentiels, une proportion de jaune plus élevée peut être considérée comme favorable du point de vue de la valeur nutritive de l'œuf. De plus, il semble que cet œuf soit apprécié localement du point de vue de la qualité gustative (Benabdeljelil et Mérat, 1992).

En conclusion, ces résultats suggèrent que le croisement expérimental testé ici n'a pas d'avantage décisif dans les conditions du présent essai, pour la viabilité et l'adaptation à l'environnement, et devrait être considérablement amélioré pour le poids moyen de l'œuf et l'efficacité alimentaire par sélection du parent Fayoumi. Sous cette condition, il pourrait valoriser sa bonne intensité de ponte et la qualité interne de ses œufs. II resterait à voir par ailleurs s'il peut présenter un avantage relatif dans des conditions d'environnement plus sévères.

\section{RÉFÉRENCES}

Benabdeljelil K, Mérat P (1992) Test de types génétiques pour une production avicole locale au Maroc. INRA Prod Anim 5, 173-178

Mérat P, Bordas A, L'Hospitalier R, Protais J, Bougon M (1983) Étude des particularités de la poule Fayoumi III. Ponte, caractéristiques des œufs, efficacité alimentaire et paramètres physiologiques de poules Fayoumi, Rhode-Island et F1 en batteries. Génét Sél Evol 15, 147-155 